\title{
STRUCTURE ELUCIDATION OF FOUR STEROIDS FROM THE SOFT CORAL SINULARIA NANOLOBATA
}

\author{
Ninh Thi Ngoc ${ }^{1,2}$, Nguyen Van Thanh ${ }^{1}$, Nguyen Xuan Cuong ${ }^{1}$, \\ Nguyen Hoai Nam ${ }^{1, *}$, Do Cong Thung ${ }^{3}$, Chau Van Minh ${ }^{1}$ \\ ${ }^{1}$ Institute of Marine Biochemistry, Vietnam Academy of Science and Technology (VAST), \\ 18 Hoang Quoc Viet, Cau Giay, Hanoi \\ ${ }^{2}$ Graduate University of Science and Technology, VAST, 18 Hoang Quoc Viet, Cau Giay, Ha Noi \\ ${ }^{3}$ Institute of Marine Environment and Resources, VAST, 246 Da Nang, Ngo Quyen, Hai Phong \\ *Email: namnguyenhoai@imbc.vast.vn
}

Received: 20 October 2016, Accepted for publication: 10 January 2017

\begin{abstract}
Four steroids namely 3 3 -hydroxyergosta-5,24(28)-diene-7-one (1), dissesterol (2), 16 $\alpha$ hydroxysarcosterol (3), and sarcophytosterol (4) were isolated from the soft coral Sinularia nanolobata using various chromatographic methods. Their structures were elucidated by detailed analysis of the 1D and 2D NMR data and comparison with the reported values.

Keywords: Sinularia nanolobata, soft coral, steroids.

\section{INTRODUCTION}

Soft corals are marine invertebrates of the order Alcyonacea, subclass Octocorallia, class Anthozoa, and phylum Cnidaria. Among these marine invertebrates, the genera Cespitularia, Clavularia, Gersemia, Lobophytum, Nephthea, Sarcophyton, and Sinularia are the most prolific [1]. The Sinularia soft corals are a rich source of hydroxylated steroids having cytotoxic effects $[2-8]$. Within the frame of our recent investigations on chemical constituents and biological activities of Vietnamese Sinularia soft corals [9, 10], we have recently reported six steroids from the soft coral Sinularia nanolobata and evaluation of their cytotoxic activity [11]. The current paper deals with detailed structure elucidation of four steroids from this species.
\end{abstract}

\section{EXPERIMENTAL}

\subsection{General methods}

The NMR spectra were recorded on a Bruker AVANCE III HD 500 spectrometer with TMS as an internal standard. Medium pressure liquid chromatography (MPLC) was carried out on a Biotage - Isolera One system. TLC was performed on Kieselgel $60 \mathrm{~F}_{254}(1.05715$; Merck) or 
RP-18 $\mathrm{F}_{254 \mathrm{~s}}$ plates. Spots were visualized by spraying with $10 \%$ aqueous $\mathrm{H}_{2} \mathrm{SO}_{4}$ solution, followed by heating for 3 - 5 minutes. Column chromatography (CC) was performed on silica gel (Kieselgel 60, 70 - 230 mesh and 230 - 400 mesh, Merck) and YMC*GEL resins (ODS-A, $12 \mathrm{~nm} \mathrm{~S}-150 \mu \mathrm{m}$, YMC Co., Ltd.).

\subsection{Biological material}

The samples of the soft coral S. nanolobata Verseveldt, 1977 were collected in Lang Co, Hue, Vietnam, in April 2015 and identified by Prof. Do Cong Thung (Institute of Marine Environment and Resources, VAST). Voucher specimens (No SN201504) were deposited at the Institute of Marine Biochemistry, VAST, Vietnam.

\subsection{Extraction and isolation}

Dried bodies of the soft coral $S$. nanolobata $(1.5 \mathrm{~kg})$ were extracted three times with methanol under ultrasonic condition. The resulting solutions were filtered, combined, and concentrated under reduced pressure to obtain the methanol residue (SNM, $140 \mathrm{~g}$ ), which was suspended in water and extracted in turn with $n$-hexane and dichloromethane resulting in extracts of $n$-hexane ( $\mathrm{SNH}, 47 \mathrm{~g}$ ), dichloromethane (SND, $5 \mathrm{~g}$ ), and an aqueous layer. Extract SNH (47 g) was crudely separated on silica gel MPLC using the mobile phase of $n$ hexane-acetone (gradient 50:1 $\rightarrow 1: 1, \mathrm{v} / \mathrm{v}$ ) to obtain six fractions, SNH1-SNH6. Fraction SNH4 $(7.3 \mathrm{~g})$ was further separated on silica gel MPLC using the mobile phase of $n$-hexane-ethyl acetate (gradient $5: 1 \rightarrow 1: 1, \mathrm{v} / \mathrm{v}$ ) to give seven subfractions, SNH4A-SNH4G. Subfraction SNH4B (2.1 g) was further separated into nine smaller fractions, SNH4B1-SNH4B9, by YMC $\mathrm{CC}$ eluting with methanol-water $(2: 1, \mathrm{v} / \mathrm{v})$. Compounds $3(5.0 \mathrm{mg})$ and $4(3.5 \mathrm{mg})$ were obtained from subfraction SNH4B7 (300 $\mathrm{mg}$ ) after purification by YMC CC eluted with acetone-water $(3: 1, \mathrm{v} / \mathrm{v})$, followed by silica gel CC with $n$-hexane-acetone $(3.5: 1, \mathrm{v} / \mathrm{v})$. Fraction SNH5 (5.0 g) was further separated on RP-18 MPLC with methanol-water $(2: 1, \mathrm{v} / \mathrm{v})$ to give six subfractions, SNH5A-SNH5G. Finally, compounds $1(6.0 \mathrm{mg})$ and $2(2.6 \mathrm{mg})$ were purified from subfraction SNH5F $(130 \mathrm{mg})$ by silica gel CC eluting with $n$-hexane-ethyl acetate $(2: 1, \mathrm{v} / \mathrm{v})$.
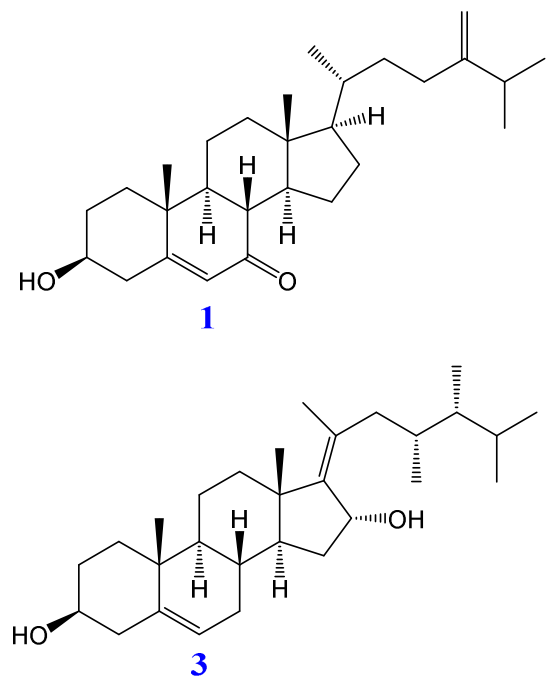

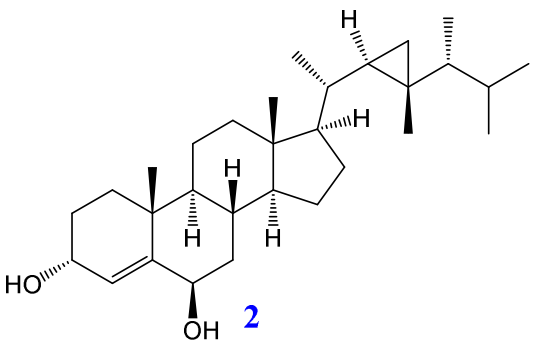

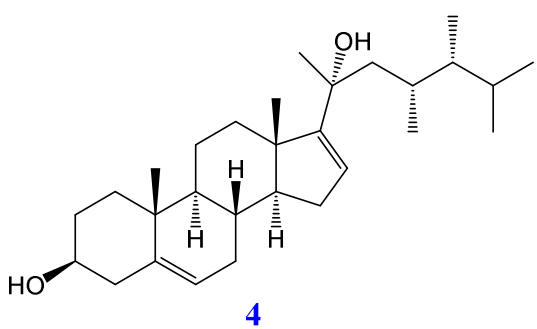

Figure 1. Chemical structures of compounds 1-4. 


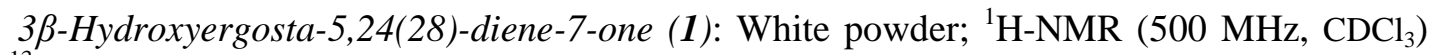
and ${ }^{13} \mathrm{C}$-NMR $\left(125 \mathrm{MHz}, \mathrm{CDCl}_{3}\right)$ see Table 1.

Dissesterol (2): White powder; ${ }^{1} \mathrm{H}-\mathrm{NMR}\left(500 \mathrm{MHz}, \mathrm{DMSO}-d_{6}\right)$ and ${ }^{13} \mathrm{C}-\mathrm{NMR}(125 \mathrm{MHz}$, DMSO- $d_{6}$ ) see Table 1.

Table 1. The NMR spectroscopic data of compounds $\mathbf{1}$ and $\mathbf{2}$.

\begin{tabular}{|c|c|c|c|c|c|c|}
\hline \multirow[b]{2}{*}{ C } & \multirow[b]{2}{*}{${ }^{\mathrm{a}} \delta_{\mathrm{C}}$} & \multicolumn{2}{|r|}{1} & \multirow[b]{2}{*}{${ }^{\mathrm{e}} \delta_{\mathrm{C}}$} & \multicolumn{2}{|r|}{2} \\
\hline & & $\delta_{C}^{b, c}$ & $\begin{array}{l}\delta_{\mathbf{H}}^{\mathbf{b}, \mathbf{d}} \\
\text { mult. }(J=\mathrm{Hz})\end{array}$ & & $\delta_{\mathrm{C}}^{\mathrm{c}, \mathrm{f}}$ & $\begin{array}{l}\delta_{\mathbf{H}}^{\mathbf{d}, \mathbf{f}} \\
\text { mult. }(J=\mathrm{Hz})\end{array}$ \\
\hline 1 & 36.34 & 36.42 & $1.18 \mathrm{~m} / 1.92 \mathrm{~m}$ & 36.5 & 36.47 & $1.20 \mathrm{~m} / 1.60 \mathrm{~m}$ \\
\hline 2 & 31.19 & 31.23 & $1.92 \mathrm{~m} / 1.60 \mathrm{~m}$ & 28.5 & 28.52 & $1.43 \mathrm{~m} / 1.78 \mathrm{~m}$ \\
\hline 3 & 70.54 & 70.55 & $3.65 \mathrm{~m}$ & 67.3 & 65.94 & $3.96 \mathrm{~m}$ \\
\hline 4 & 41.81 & 41.89 & $2.38 \mathrm{~m} / 2.49 \mathrm{~m}$ & 128.1 & 128.40 & $5.37 \mathrm{br} \mathrm{s}$ \\
\hline 5 & 165.07 & 165.34 & - & 147.1 & 145.17 & - \\
\hline 6 & 126.13 & 126.14 & $5.66 \mathrm{~s}$ & 73.6 & 72.10 & $4.02 \mathrm{br} \mathrm{s}$ \\
\hline 7 & 202.30 & 202.45 & - & 38.9 & 39.33 & $1.15 \mathrm{~m} / 1.98 \mathrm{~m}$ \\
\hline 8 & 45.41 & 45.48 & $2.22 \mathrm{~m}$ & 30.1 & 29.72 & $1.82 \mathrm{~m}$ \\
\hline 9 & 49.95 & 49.98 & $1.32 \mathrm{~m}$ & 54.1 & 53.96 & $0.68 \mathrm{~m}$ \\
\hline 10 & 38.28 & 38.36 & - & 36.7 & 36.04 & - \\
\hline 11 & 21.22 & 21.28 & $1.54 \mathrm{~m} / 1.55 \mathrm{~m}$ & 20.8 & 20.29 & $1.35 \mathrm{~m} / 1.45 \mathrm{~m}$ \\
\hline 12 & 38.69 & 38.76 & $1.12 \mathrm{~m} / 2.01 \mathrm{~m}$ & 39.7 & 39.93 & $1.01 \mathrm{~m} / 1.79 \mathrm{~m}$ \\
\hline 13 & 43.14 & 43.21 & - & 42.8 & 42.28 & - \\
\hline 14 & 49.90 & 50.02 & $1.50 \mathrm{~m}$ & 55.8 & 55.35 & $0.96 \mathrm{~m}$ \\
\hline 15 & 26.31 & 26.38 & $2.39 \mathrm{~m} / 1.26 \mathrm{~m}$ & 24.2 & 23.66 & $1.08 \mathrm{~m} / 1.58 \mathrm{~m}$ \\
\hline 16 & 28.54 & 28.60 & $1.28 \mathrm{~m} / 1.89 \mathrm{~m}$ & 28.0 & 27.42 & $1.35 \mathrm{~m} / 1.96 \mathrm{~m}$ \\
\hline 17 & 54.63 & 54.70 & $1.06 \mathrm{~m}$ & 57.8 & 57.33 & $1.25 \mathrm{~m}$ \\
\hline 18 & 12.00 & 12.05 & $0.66 \mathrm{~s}$ & 11.8 & 11.56 & $0.69 \mathrm{~s}$ \\
\hline 19 & 17.33 & 17.38 & $1.17 \mathrm{~s}$ & 21.1 & 20.50 & $1.17 \mathrm{~s}$ \\
\hline 20 & 35.66 & 35.72 & $1.15 \mathrm{~m}$ & 35.1 & 33.95 & $1.08 \mathrm{~m}$ \\
\hline 21 & 18.87 & 18.94 & $0.93 \mathrm{~d}(7.0)$ & 21.1 & 20.53 & $0.98 \mathrm{~d}(7.0)$ \\
\hline 22 & 34.68 & 34.75 & $1.53 \mathrm{~m}$ & 31.8 & 31.35 & $0.22 \mathrm{~m}$ \\
\hline 23 & 30.97 & 31.05 & $1.84 \mathrm{~m} / 2.08 \mathrm{~m}$ & 25.6 & 25.00 & - \\
\hline 24 & 156.79 & 156.83 & - & 50.6 & 49.62 & $0.32 \mathrm{~m}$ \\
\hline 25 & 33.76 & 33.82 & $2.20 \mathrm{~m}$ & 32.0 & 31.08 & $1.59 \mathrm{~m}$ \\
\hline 26 & 21.87 & 22.08 & $0.99 \mathrm{~d}(7.0)$ & 21.2 & 20.45 & $0.85 \mathrm{~d}(7.0)$ \\
\hline 27 & 22.01 & 21.93 & $1.00 \mathrm{~d}(7.0)$ & 21.8 & 21.60 & $0.94 \mathrm{~d}(7.0)$ \\
\hline 28 & 106.03 & 106.10 & $4.69 \mathrm{br} \mathrm{s} / 4.62 \mathrm{br} \mathrm{s}$ & 15.2 & 14.31 & $0.92 \mathrm{~d}(7.0)$ \\
\hline 29 & & & & 14.0 & 13.82 & $0.91 \mathrm{~s}$ \\
\hline 30 & & & & 20.9 & 20.12 & $-0.10 \mathrm{dd}(4.5,5.5)$ \\
\hline & & & & & & $0.45 \mathrm{dd}(4.5,9.0)$ \\
\hline
\end{tabular}

${ }^{\mathrm{a}} \delta_{\mathrm{C}}$ of $3 \beta$-hydroxyergosta-5,24(28)-diene-7-one [12], ${ }^{\mathrm{b}}$ recorded in $\mathrm{CDCl}_{3},{ }^{\mathrm{c}} 125 \mathrm{MHz},{ }^{\mathrm{d}} 500 \mathrm{MHz},{ }^{\mathrm{e}} \delta_{\mathrm{C}}$ of

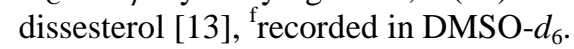

\section{RESULTS AND DISCUSSION}

Compound 1 was obtained as a white powder. The NMR features indicated an ergosterol derivative, one main constituent of Sinularia soft corals [2]. The ${ }^{1} \mathrm{H}-$ and ${ }^{13} \mathrm{C}-\mathrm{NMR}$ spectra of 1 showed typical signals of one oxymethine $\left[\delta_{\mathrm{C}} 70.55(\mathrm{C}-3) / \delta_{\mathrm{H}} 3.65(1 \mathrm{H}, \mathrm{m}, \mathrm{H}-3)\right]$, one trisubstituted endocyclic double bond $\left[\delta_{\mathrm{C}} 165.34(\mathrm{C}, \mathrm{C}-5)\right.$ and $126.14(\mathrm{CH}, \mathrm{C}-6) / \delta_{\mathrm{H}} 5.66(1 \mathrm{H}, \mathrm{s}$, 
H-6)], one ketone [ $\delta_{\mathrm{C}} 202.45(\mathrm{C}, \mathrm{C}-7)$ ], one 1,1-disubstituted double bond [ $\delta_{\mathrm{C}} 156.83(\mathrm{C}, \mathrm{C}-24)$ and $106.10\left(\mathrm{CH}_{2}, \mathrm{C}-28\right) / \delta_{\mathrm{H}} 4.62$ and $4.69(\mathrm{H}-28)$, each $1 \mathrm{H}, \mathrm{br}$ s], two tert-methyls $\left[\delta_{\mathrm{C}} 12.05(\mathrm{C}-\right.$ $18)$ and $17.38(\mathrm{C}-19) / \delta_{\mathrm{H}} 0.66(\mathrm{H}-18)$ and $1.17(\mathrm{H}-19)$, each $\left.3 \mathrm{H}, \mathrm{s}\right]$, and three sec-methyls $\left[\delta_{\mathrm{C}}\right.$ $18.94(\mathrm{C}-21), 22.08(\mathrm{C}-26)$, and $21.93(\mathrm{C}-27) / \delta_{\mathrm{H}} 0.93(\mathrm{H}-21), 0.99(\mathrm{H}-26)$, and $1.00(\mathrm{H}-27)$, each $3 \mathrm{H}, \mathrm{d}, J=7.0 \mathrm{~Hz}$. The HMBC correlations (Fig. 2) of H-6 with C-4, C-8, and C-10; H-8 with C-7, and H-19 with C-1, C-5, C-9, and C-10, clearly confirmed positions of the double bond C5/C-6 and ketone C-7. The detailed analysis of other HMBC correlations and an agreement of the ${ }^{13} \mathrm{C}$-NMR chemical shifts of $\mathbf{1}$ with the reported data led to assignment of $\mathbf{1}$ as $3 \beta$ hydroxyergosta-5,24(28)-diene-7-one [12].
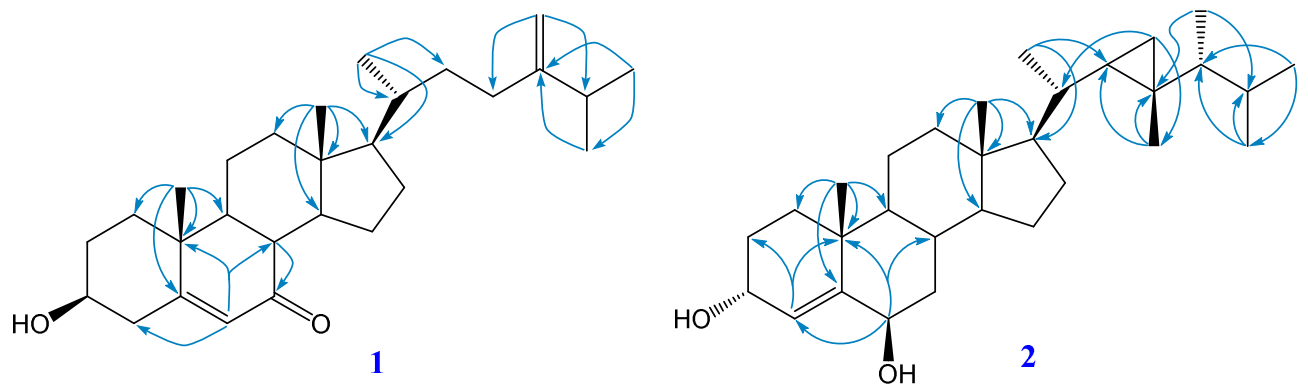

Figure 2. Key HMBC correlations of compounds $\mathbf{1}$ and 2.

Compound 2 was also obtained as a white powder. The ${ }^{13} \mathrm{C}-\mathrm{NMR}$ and HSQC spectra confirmed 30 carbon signals including seven methyls, eight methylenes, eleven methines, and four quaternary carbons. The signals at $\delta_{\mathrm{C}} 11.56$ (C-18), 20.50 (C-19), 20.53 (C-21), 20.45 (C26), 21.60 (C-27), 14.31 (C-28), and 13.82 (C-29) indicated the presence of seven methyl groups. Moreover, two oxymethine groups $\left[\delta_{\mathrm{C}} 65.94(\mathrm{C}-3)\right.$ and $\left.72.10(\mathrm{C}-6)\right]$ and a trisubstituted double bond $\left[\delta_{\mathrm{C}} 128.40(\mathrm{CH}, \mathrm{C}-4)\right.$ and $\left.145.17(\mathrm{C}, \mathrm{C}-5)\right]$ were also identified. In the ${ }^{1} \mathrm{H}-\mathrm{NMR}$ spectrum, the presence of four high-field protons at $\delta_{\mathrm{H}} 0.22(1 \mathrm{H}, \mathrm{m}, \mathrm{H}-22), 0.32(1 \mathrm{H}, \mathrm{m}, \mathrm{H}-24)$, $-0.10\left(1 \mathrm{H}, \mathrm{dd}, J=4.5,5.5 \mathrm{~Hz}, \mathrm{H}_{\beta}-30\right)$, and $0.45\left(1 \mathrm{H}, \mathrm{dd}, J=4.5,9.0 \mathrm{~Hz}, \mathrm{H}_{\alpha}-30\right)$ is characteristic of a gorgosterol-type side chain possessing a cyclopropane ring [14]. The HMBC cross peaks of H-19 $\left(\delta_{\mathrm{H}} 1.17\right)$ with C-1 $\left(\delta_{\mathrm{C}} 36.47\right) / \mathrm{C}-5\left(\delta_{\mathrm{C}} 145.17\right) / \mathrm{C}-9\left(\delta_{\mathrm{C}} 53.96\right) / \mathrm{C}-10\left(\delta_{\mathrm{C}}\right.$ $36.04)$ and H-6 $\left(\delta_{\mathrm{H}} 4.02\right)$ with C-4 $\left(\delta_{\mathrm{C}} 128.40\right) / \mathrm{C}-8\left(\delta_{\mathrm{C}} 29.72\right) / \mathrm{C}-10\left(\delta_{\mathrm{C}} 36.04\right)$ confirmed positions of the double bond at $\mathrm{C}-4 / \mathrm{C}-5$ and the hydroxy group at $\mathrm{C}-6$. The detailed analyses of other HMBC correlations (Fig. 2) and the comparison of the ${ }^{13} \mathrm{C}-\mathrm{NMR}$ chemical shifts of 2 with those reported (Table 1) clearly identified compound $\mathbf{2}$ as dissesterol [13].

The other compounds were elucidated as 16 $\alpha$-hydroxysarcosterol (3) [15] and sarcophytosterol (4) [16] by detailed analysis of their 1D and 2D NMR data and in comparison with reported data.

Acknowledgements. This research is funded by Vietnam National Foundation for Science and Technology Development (NAFOSTED) under grant number 104.01-2013.31. The authors are grateful to MSc Dang $\mathrm{Vu}$ Luong, Institute of Chemistry, VAST for measurement of the NMR spectra.

\section{REFERENCES}

1. Liang, X. T., Fang, W. S. - Medicinal Chemistry of Bioactive Natural Products. John Wiley \& Sons, New Jersey, 2006, pp. 257-300. 
2. Lakshmi, V., Kumar, R. - Metabolites from Sinularia species. Nat Prod Res 23 (2009) 801-850.

3. Chao, C. H., Chou, K. J., Huang, C. Y., Wen, Z. H., Hsu, C. H., Wu, Y. C., Dai, C. F., Sheu, J. H. - Steroids from the soft coral Sinularia crassa. Mar Drugs 10 (2012) 439-450.

4. Huang, C. Y., Su, J. H., Duh, C. Y., Chen, B. W., Wen, Z. H., Kuo, Y. H., Sheu, J. H. - A new 9,11-secosterol from the soft coral Sinularia granosa. Bioorg Med Chem Lett 22 (2012) 4373-4376.

5. Li, R., Shao, C. L., Qi, X., Li, X. B., Li, J., Sun, L. L., Wang, C. Y. - Polyoxygenated sterols from the South China Sea soft coral Sinularia sp. Mar Drugs 10 (2012) 1422-1432.

6. Aboutabl el, S. A., Azzam, S. M., Michel, C. G., Selim, N. M., Hegazy, M. F., Ali, A. H., Hussein, A. A. - Bioactive terpenoids from the Red Sea soft coral Sinularia polydactyla. Nat. Prod. Res. 27 (2013) 2224-2226.

7. Tseng, Y. J., Wang, S. K., Duh, C. Y. - Secosteroids and norcembranoids from the soft coral Sinularia nanolobata. Mar. Drugs 11 (2013) 3288-3296.

8. Yen, W. H., Chen, W. F., Cheng, C. H., Dai, C. F., Lu, M. C., Su, J. H., Su, Y. D., Chen, Y. H., Chang, Y. C., Chen, Y. H., Sheu, J. H., Lin, C. H., Wen, Z. H., Sung, P. J. - A new $5 \alpha, 8 \alpha$-epidioxysterol from the soft coral Sinularia gaweli. Molecules 18 (2013) 28952903.

9. Ngoc, N. T., Huong, P. T. M., Diep, C. N., Thanh, N. V., Thung, D. C., Cuong, N. X., Nam, N. H., Minh, C. V. - Steroids isolated from the soft coral Sinularia microspiculata. Vietnam J. Chem. 54 (2016) 82-85.

10. Ngoc, N. T., Diep, C. N., Thanh, N. V., Thung, D. C., Cuong, N. X., Nam, N. H., Minh, C. V. - Sesquiterpenes isolated from the soft coral Sinularia microspiculata. Vietnam J. Chem. 53 (2015) 14-17.

11. Ngoc, N. T., Huong, P. T. M., Thanh, N. V., Cuong, N. X., Nam, N. H., Thung, D. C., Kiem, P. V., Minh, C. V. - Steroid constituents from the soft coral Sinularia nanolobata. Chem. Pharm. Bull. 64 (2016) 1417-1419.

12. Guerriero, A., D'Ambrosio, M., Pietra, F., Debitus, C., Ribes, O. - Pteridines, sterols, and indole derivatives from the lithistid sponge Corallistes undulatus of the coral sea. J. Nat. Prod. 56 (1993) 1962-1970.

13. Thao, N. P., Nam, N. H., Cuong, N. X., Tai, B. H., Quang, T. H., Ngan, N. T. T., Luyen, B. T. T., Yang, S. Y., Choi, C. H., Kim, S., Chae, D., Koh, Y.-S., Kiem, P. V., Minh, C. V., Kim, Y. H. - Steroidal constituents from the soft coral Sinularia dissecta and their inhibitory effects on lipopolysaccharide-stimulated production of pro-inflammatory cytokines in bone marrow-derived dendritic cells. Bull. Korean Chem. Soc. 34 (2013) 949-952.

14. D'Armas, H. T., Mootoo, B. S., Reynolds, W. F. - Steroidal compounds from the Caribbean octocoral Eunicea laciniata. J. Nat. Prod. 63 (2000) 1669-1671.

15. Thanh, N. V., Ngoc, N. T., Anh, H. L. T., Thung, D. C., Thao, D. T., Cuong, N. X., Nam, N. H., Kiem, P. V., Minh, C. V. - Steroid constituents from the soft coral Sinularia microspiculata. J Asian Nat. Prod. Res. 18 (2016) 938-944.

16. Lu, Y., Lin, Y.-C., Wen, Z.-H., Su, J.-H., Sung, P.-J., Hsu, C.-H., Kuo, Y.-H., Chiang, M. Y., Dai, C.-F., Sheu, J.-H. - Steroid and cembranoids from the Dongsha atoll soft coral Lobophytum sarcophytoides. Tetrahedron 66 (2010) 7129-7135. 